Ebisu Ebisu

Études japonaises Études japonaises

47 | printemps-été 2012

Catastrophes du 11 mars 2011, désastre de

Fukushima : fractures et émergences

\title{
Technologie souveraine et contrôle démocratique
}

Sovereign Technology and Democratic Control

君臨するテクノロジーと民主的コントロール

\section{Alain-Marc Rieu}

\section{(2) OpenEdition}

Journals

Édition électronique

URL : http://journals.openedition.org/ebisu/265

DOI : 10.4000/ebisu.265

ISSN : 2189-1893

Éditeur :

Institut français de recherche sur le Japon (UMIFRE 19 MAEE-CNRS), Maison franco-japonaise

Édition imprimée

Date de publication : 1 juin 2012

Pagination : 69-78

ISSN : 1340-3656

Référence électronique

Alain-Marc Rieu, «Technologie souveraine et contrôle démocratique », Ebisu [En ligne], 47 | printempsété 2012, mis en ligne le 03 avril 2014, consulté le 19 avril 2019. URL : http://journals.openedition.org/ ebisu/265 ; DOl : 10.4000/ebisu.265

(c) Institut français de recherche sur le Japon à la Maison franco-japonaise 


\title{
Technologie souveraine et contrôle démocratique
}

\author{
Alain-Marc RIEU
}

\section{Une catastrophe institutionnelle}

Fukushima est, et restera, le nom d'une catastrophe humaine et écologique mais aussi institutionnelle et politique. Dans le contexte de déconstruction dans lequel est engagé le Japon depuis les années 1990, la reconstruction sera un échec de plus si elle n'est pas une refondation. Tous les Japonais savent d'expérience qu'une refondation est toujours politique. Mais ils ont en commun une même expérience : depuis les premières années de Meiji, depuis le début de l'ère Taishō et surtout depuis les années 1945-1950 et le projet confisqué d'une "démocratie d'après guerre ", la fonction politique est atrophiée, une attente indéfiniment suspendue, un projet encore à venir ${ }^{1}$. Il suffit d'étudier l'évolution du Japon depuis les années 1990 en y incluant la réponse à la catastrophe de mars 2011 : indécision des

Alain-Marc Rieu (amrieu[at]gmail.com) est professeur de philosophie contemporaine et d'épistémologie (Science Studies) à l'université de Lyon-Jean Moulin, membre de l'Institut d'Asie orientale à l'ENS de Lyon (<http://w7.ens-lyon.fr/amrieu/>).

1. Sur les conditions dans lesquelles les institutions politiques actuelles ont été conçues, voir Dower (1999), chapitre 13. L'idée d'une "modernité inachevée " traverse l'œuvre de Maruyama Masao : "Loin d'avoir dépassé la pensée moderne, nous, au Japon, ne sommes pas encore parvenus à l'achever complètement " (Maruyama 1946). C'est l'idée directrice d'une "démocratie d'après guerre ", dérobée au peuple japonais, source d'un débat fondamental et toujours actuel sur la démocratie, la subjectivité et la société civile : voir Barshay (2004), Ōe (1999), Rieu (1998, 2001). 
gouvernements successifs, débats sans fin, réformes différées, conflits entre les partis et les factions des partis pour d'abord neutraliser les projets, puis leur adoption, enfin leur mise en œuvre, premiers ministres se succédant sans fin, partis d'opposition manipulés par d'anciens membres de l'autre camp, ralliements soudains et défections aussi rapides, corruption rampante par jeux d'influence, financements politiques occultes. L'atrophie de la fonction politique crée un climat permanent d'incertitude et même d'angoisse dans la population, dans l'industrie et le commerce, jusque dans les universités et la recherche. L'atrophie politique fragilise tout le pays.

La pratique du tenko 転向 (revirement), une constante de l'histoire japonaise ${ }^{2}$, en est la preuve. Un bel exemple est le tenkō effectué par Kan Naoto, président du Parti démocratique du Japon et Premier ministre de juin 2010 à août 2011 : après avoir affirmé que la "croissance verte » était un élément clef du "changement de régime " adopté par la majorité des Japonais le 30 août 2009, un haut responsable du METI annonçait le 2 décembre 2010 que la politique énergétique du Japon ne s'inscrivait plus dans les objectifs du Protocole de Kyoto. Cette déclaration était confirmée le lendemain par le bureau du Premier ministre. Il avait cédé " aux intérêts des industries monopolisant la production d'électricité (dont Tepco) et des industries productrices de carbone comme le ciment et l'acier, ainsi que des diverses industries en dépendant ${ }^{3} »($ DeWit $\&$ Iida 2011). Plus révélateur encore est le nouveau tenkō qu'accomplit le Premier ministre Kan après la catastrophe, le 14 juillet 2011 en se prononçant pour " la réalisation d'une société fonctionnant sans le nucléaire ». Comment expliquer ce revirement ? Simplement en citant le titre d'un article du journal Le Monde du 2 août 2011 : «Au Japon, le financement des partis par les compagnies d'électricité fait scandale ». Le Premier ministre en question se trouvant alors sur le point de quitter ses fonctions, ce tenkō n'engageait en fait que

2. Le revirement n'est pas une conversion volontaire mais le résultat d'un rapport de force : «La conversion ne ressemble qu'en apparence au tenkō car elle est de sens inverse. Si le tenkō est un mouvement vers l'extérieur, la conversion est un mouvement vers l'intérieur, la subjectivité. La conversion se produit pour préserver le soi alors que le tenkō se produit en renonçant au soi. » (Takeuchi 2004 : 75).

3. Voir le commentaire officiel de la Fédération des compagnies électriques du Japon sur la victoire du Parti démocrate du Japon le 31 août 2010, un avertissement sinon une menace : <http://www.fepc.or.jp/english/news/message/1199866_1653.html>. 
lui ${ }^{4}$. Malgré les attaques qu’il subit, le pouvoir nucléaire ne renonça pas à ses objectifs. Certes, un consensus s'est formé sur le développement rapide des énergies vertes. Mais le nucléaire doit assurer la transition entre les deux régimes énergétiques, ce qui lui donne le temps de se refaire une vertu et d'opérer sa conversion.

\section{Fukushima : le pouvoir mis à nu}

Le pouvoir qui pilote le Japon n'est pas réductible à la bureaucratie. La prendre pour bouc émissaire des malheurs du Japon dissimule le conglomérat d'intérêts au pouvoir. Ce pouvoir n'est pas caché mais au contraire bien visible. Au Japon, tout le monde perçoit les réseaux qui associent les grands groupes industriels, les factions politiques, les anciens des grandes universités, les associations patronales et les syndicats, les responsables des principaux ministères, des instituts de recherche, des lobbies et think tanks, y compris les grands journaux historiques qui prétendent au nom de l'opinion publique exprimer l'identité ou l'intérêt national. Certes, on ne perçoit pas tous les détails, mais il suffit d'observer pour comprendre. Il suffit de tirer les fils d'une affaire ${ }^{5}$ ou d'une catastrophe pour que se révèlent des pans entiers d'une société. Combien d'hommes politiques tombent (et reviennent) chaque année pour corruption ? Combien de projets inutiles et même dangereux ? Les réformes, les ministres se succèdent comme les

4. Kan Naoto ne semble pas vouloir rester seul : il a rassemblé une trentaine de députés de son parti, le PD, pour créer une commission de réflexion sur la sortie du nucléaire le 28 mars 2012 (Datsu genpatsu : rōdo mappu o kangaeru kai 脱原発・ロードマップを考 える会). Son but est de freiner le projet de l'actuel gouvernement Noda de redémarrer la puissante centrale nucléaire d'Ōi 大飯 dans le Kansai. Kan Naoto veut prendre pour modèle la feuille de route de sortie du nucléaire adoptée en Allemagne. Par ailleurs, un comité supra-partisan (Genpatsu zero no kai 原発ゼロの会) comprenant des députés du PD, du PLD et du Minna no tō ainsi que du Parti social-démocrate a été créé le 27 mars 2012, dont les objectifs sont la sortie totale du nucléaire, le refus de tout recyclage des déchets nucléaires et l'adoption d'un grand tournant vers les énergies renouvelables [N.D.L.R., le 3 avril 2012].

5. C'était la méthode de Chalmers Johnson, par exemple pour l'affaire Tanaka. Il analyse le concept japonais de "corruption structurelle " (közō oshoku 構造污職) (Johnson 1995 : 184) pour lui opposer une « analytique du pouvoir ». Voir Rieu (2001). 
personnages d'une pièce de théâtre. Les citoyens japonais ne sont pas dupes - même s'ils continuent de voter. Le Japon n'est pas une exception : ce désenchantement politique se retrouve dans tous les pays démocratiques. Au moins le pouvoir y est-il (comme en Angleterre ou aux États-Unis) plus visible qu'ailleurs, qu'en France en particulier.

Et puis il y eut la catastrophe nucléaire de Fukushima le 11 mars 2011, qui dure encore et durera longtemps : populations déplacées et exposées aux radiations, mal informées et manipulées, terres, océan, cours d'eau, récoltes, bétail contaminés, régions devenues inhabitables, incultivables pour une durée indéterminée, sans être certain de la fiabilité des informations officielles. Fukushima est le grand analyseur dont avait peur la société japonaise. Toutes les craintes sur la manipulation, la désinformation, l'incompétence, la primeur de l'intérêt du pouvoir sur l'intérêt collectif ont été vérifiées. La confiance est désormais impossible. L'exemple le plus révélateur est le refoulement de la différence entre les dégâts commis par le tremblement de terre et ceux commis par le tsunami, 45 minutes plus tard. Si les destructions ayant enclenché la fusion du cœur du réacteur furent causées par le tremblement de terre, alors toutes les centrales du même type que Fukushima sont dangereuses et doivent être fermées, par exemple celle de Hamaoka dans le département de Shizuoka construite sur une faille (McNeill \& Adelstein 2011, Koide 2011). Si le tsunami est la cause de la catastrophe nucléaire de Fukushima, contrairement à ce que prétend Tepco, sa culpabilité n'est pas moindre. Elle est même plus grande puisque les dirigeants de Tepco ont choisi de ne pas concevoir, ni d'adapter l'installation au risque probable d'un tsunami dans une région. Ce risque d'une déferlante de plus de dix mètres de haut était reconnu depuis au moins 2008, Tepco a fait le choix de le minorer. Or Tepco n'a pas pu faire un tel choix sans le consentement (explicite ou tacite) et la couverture d'une technostructure qui trouvait dans l'industrie nucléaire une justification de ses présupposés, ses intérêts et ses valeurs (Shiokura 2011). La leçon vaut pour tous les pays possédant une industrie nucléaire, la France en premier. 


\section{Le nucléaire : une technologie souveraine}

La vraie contamination est la technostructure elle-même : elle est responsable de la catastrophe. Elle l'a rendue possible en sous-estimant et en déniant les risques encourus par la population, en n'adoptant pas les mesures de secours et de sécurité nécessaires, en déniant la compétence des ingénieurs qui dénonçaient les défauts de l'installation et les dangers en résultant. Les causes d'une telle prise de risque sont économiques : réduire de tels risques est d'un coût lui-même si élevé qu'un tel projet n'est pas rentable, n'aurait pas été entrepris ou aurait été abandonné sans le soutien d'une technostructure au pouvoir. Parce qu'elles engagent la société tout entière, les industries souveraines tendent nécessairement à reproduire dans leur organisation et leur fonctionnement le modèle institutionnel qui les développe et dont elles sont indissociables (Nishioka 2011 ; Hecht 2004). On ne peut omettre les liens étroits entre le nucléaire civil et militaire, constitutionnellement bloqués au Japon. Parce qu'elle a pour but d'assurer l'indépendance énergétique de la nation, l'industrie nucléaire jouit d'un statut spécial en tant qu'expression et instrument de souveraineté. Toute l'industrie nucléaire est donc une affaire d'État, par là même dangereuse parce qu'incontrôlable par des institutions démocratiques. Le risque intrinsèque de la technologie nucléaire est en réalité un problème secondaire par rapport à l'impossible contrôle démocratique des installations et des industries qui les exploitent. Un autre cas typique est la France. Cela signifie qu'une production nucléaire d'énergie pourrait éventuellement être débattue une fois les conditions institutionnelles d'évaluation et de surveillance établies, démocratiquement garanties.

L'autre cause de la catastrophe est aussi grave : plus un pouvoir se sent puissant, fort des intérêts agrégés, légitime dans ses objectifs, plus il est prêt à prendre des risques, à croire que l'intérêt qu'il incarne ne contredit pas les intérêts et même l'existence de la population qu'il administre. Tout pouvoir tend vers un seuil de contre productivité qui le rend dangereux. Plus un pouvoir tend vers ce seuil, plus il devient fragile et concentre alors les moyens de se protéger. À lire ce que les médias nous ont appris, la technostructure est mise à nu par la catastrophe de Fukushima aussi bien que par la construction de centrales dans des zones hautement sismiques. La technostructure est détruite autant que la centrale de Fukushima. Pourtant, c'est elle qui tente pendant le printemps et depuis l'été 2011 de réduire 
ou gommer sa responsabilité en organisant et pilotant la reconstruction conformément à ses préconceptions, ses méthodes et critères, ses finalités et ses intérêts. Elle a pourtant perdu toute légitimité et crédibilité.

Cette situation crée un contexte institutionnel et politique nouveau. En effet, l'échec final de la technostructure au pouvoir depuis les années 1980 ne signifie pas que, pris séparément, ses composants soient incompétents, inutiles et dangereux pour la population. Certes l'appareil d'État a ses propres intérêts, mais son but n'est pas d'asservir la population. Le problème réside dans l'emboîtement des composants de cette technostructure. Ce pouvoir réticulé détourne et monopolise la fonction politique à son profit en marginalisant d'autres composants du système social, essentiellement les jeunes générations, le monde des employés aussi bien que les intellectuels, les milieux de la recherche, les retraités, ceux qui vivent aussi bien dans les campagnes que dans les périphéries du pouvoir. Actuellement, la technostructure exclut la participation effective de la population, ce peuple japonais au nom duquel elle prétend agir et satisfaire l'intérêt national. La technostructure neutralise la fonction politique : les partis s'affrontent et les gouvernements se succèdent comme si les conflits qui les animent exprimaient les besoins de la population. Au pire moment de la catastrophe, les partis politiques et les factions de chaque parti ont montré qu'ils ne savaient pas faire autre chose que régler leurs conflits internes en croyant traiter ainsi des problèmes auxquels étaient confrontés ceux qui les élisent et en attendent des solutions. Les partis jouent à gouverner les uns après les autres, comme un manège qui ne touche pas le sol.

Or une technostructure ne gouverne pas; elle contrôle, oriente et pilote une économie et une société en contraignant ou conseillant un gouvernement. Pourquoi une technostructure a-t-elle besoin d'un gouvernement ? Pour se dissimuler dans son ombre ? Comme gage de démocratie ? La réponse est évidente : comme médiation envers ce qu'elle exclut de ses réseaux : le peuple, la population en général, tous ceux qui travaillent, consomment et élèvent des enfants. Ils votent aussi. La situation actuelle est claire : la technostructure étant déconstruite, la seule façon de répondre à la situation présente est d'imaginer et établir d'autres interactions entre les pôles de pouvoir que sont l'administration d'État, le monde industriel, les médias, les institutions de recherche et d'enseignement, entre ces pôles qui pilotent l'évolution du Japon. Les victimes de la déconstruction, de la crise et de la catastrophe conjuguées ne peuvent pas être refoulées : elles 
sont objectivement devenues un pôle de pouvoir qui reconfigure la technostructure invalidée. La transition s'est creusée et accélérée, un seuil virtuel de recomposition est atteint.

\section{Le nucléaire ou la démocratie}

Reste à penser un tel seuil pour lui donner forme. Le Japon dispose d'une solide expérience en ce domaine puisque s'y pratique depuis presque vingt ans ce que Michel Callon (2001), qui s’y réfère, a nommé une « démocratie technique ". Ces débats sont organisés selon des méthodes dont la rigueur est reconnue (ScienceWise 2011) : ils présentent un double avantage, à la fois former et informer la population selon un processus de capillarité ; et, d'autre part, informer les agences en charge de la conception, du financement et de la gestion des politiques de recherche. Ces débats et ces expériences permettent à la fois de comprendre les compétences et les demandes qu'exprime l'opinion publique, et de la former pour l'inscrire dans le débat démocratique afin d'ouvrir et d'étendre le processus politique de décision. C'est une avancée démocratique majeure. On est loin des invocations rhétoriques de la société civile. Les spécialistes japonais disposent des méthodes et des compétences leur permettant d'analyser et de mettre en forme aussi bien les besoins que les désirs des populations victimes du tsunami et de la catastrophe nucléaire de Fukushima. Ces populations disposent des connaissances tacites et des compétences explicites pouvant être prises en compte dans le processus de reconstruction. C'est pourquoi le comportement du ministre de la Reconstruction, Matsumoto Ryū, nommé le 27 juin 2011, démissionné le 5 juillet, est apparu doublement scandaleux : son attitude exprimait l'arrogance du pouvoir établi mais aussi l'aveuglement de ce pouvoir face à la catastrophe ; non seulement il était incapable de reconnaître la compétence des responsables locaux, mais aussi de reconnaître les compétences disponibles au Japon permettant d'inscrire les savoirs tacites de la population dans la décision politique. C'est ce que montrent au quotidien ces mères de famille inquiètes pour la santé de leurs enfants, équipées de compteurs Geiger, qui dressent sur Internet des cartes de la radioactivité des sols, qui interpellent les responsables politiques locaux et nationaux pour exiger des solutions, qui les trouvent si les autorités en sont incapables. $\mathrm{Ni}$ la bureaucratie, ni les élus ne pourront faire rentrer ces femmes dans 
leurs foyers : elles sont entrées dans l'espace public et elles y resteront. Elles savent ce que signifie la politique pour un peuple.

Ce raisonnement conduit à une conclusion : les recherches et les débats sur la sécurité nucléaire, la fiabilité et la sécurité des centrales, sont vains. Ils sont en effet infinis et jamais conclusifs. Ils entretiennent une ambiguïté permettant à l'industrie nucléaire et aux milieux économiques et politiques qui lui sont associés de poursuivre pendant la durée du débat leur développement en affirmant qu'ils maîtrisent les risques, qu'ils respectent le principe de précaution, qu'ils font tout ce qui est en leur possible pour protéger les populations et assurer un approvisionnement fiable. La catastrophe de Fukushima change les conditions du débat : elle établit que les institutions démocratiques actuelles ne sont pas capables de contrôler et de gérer un agencement techno-industriel souverain qui suppose une telle agrégation de capital, d'industries associées et d'impact sur la vie politique, sociale et économique d'une nation qu'il excède tout contrôle démocratique. Il est de fait impossible de prendre position sur le risque des centrales nucléaires actuelles et celles de demain. Peut-être la technologie est-elle maîtrisable et le sera-t-elle. Peut-être des nations, des régions accepteront-elles de développer cette technologie. On ne peut pas l'exclure. En effet, nous ne sommes pas seulement en situation de risque mais aussi d'incertitude. Une chose au moins est certaine : les pays industriels capables de développer une telle technologie et son industrie ne disposent pas actuellement des institutions politiques nécessaires pour gérer aussi bien le risque que l'incertitude. Comme le montre Fukushima, comme l'a montré Tchernobyl, le risque premier, le plus dangereux, est politique et institutionnel. La question énergétique suppose donc une avancée démocratique majeure. La pensée politique et l'épistémologie doivent (enfin) se remettre en mouvement et apprendre à converger. Michel Callon a ouvert le chemin et l'a balisé. La tâche est difficile car les résistances sont fortes. Mais on peut déjà en déduire une chose : avant qu'on ait conçu de nouvelles institutions démocratiques et qu'on les ait effectivement mises en œuvre, tant d'années auront passé qu'entre temps de multiples énergies alternatives seront au point et rentables. Ainsi l'industrie nucléaire, héritage du siècle passé, n’a pas d'avenir. Elle n'est qu'un pouvoir au présent. 


\section{Références}

Les documents en anglais sur la catastrophe de Fukushima sont disponibles sur le site The Asia-Pacific Journal-Japan Focus : <http://www.japanfocus.org/home>.

\section{BARSHAY Andrew, 2004}

The Social Sciences in Modern Japan. The Marxian and Modernist Traditions, Berkeley, University of California Press, chapitre 7 «Imagining democracy in post-war Japan ».

\section{CALLON Michel, LASCOUMES Pierre \& BARTHE Yannick, 2001}

Agir dans un monde incertain. Essai sur la démocratie technique, Paris, Seuil.

DeWIT Andrew \& IIDA Tetsunari, 2011 "The "Power Elite" and EnvironmentalEnergy Policy in Japan », The Asia-Pacific Journal, Vol 9, Issue 4 No 4, January 24.

DOWER John, 1999

Embracing Defeat. Japan in the Wake of World War II, New York, Norton.

\section{HECHT Gabrielle, 2004}

Le rayonnement de la France. Énergie nucléaire et identité nationale après la Seconde Guerre mondiale, Paris, Éditions de la découverte.

\section{JOHNSON Chalmers, 1995}

«Tanaka Kakuei, Structural Corruption and the Advent of the Machine Politics in Japan ", Japan: Who Governs?

The Rise of the Development State, New York, Norton.

\section{KOIDE Hiroaki, 2011}

"The Truth About Nuclear Power: Japanese Nuclear Engineer Calls for Abolition », trad. par SAKAI Yasuyuki et NORIMATSU Satoko, The Asia-Pacific Journal, Vol 9, Issue 31 No 5, August 1.

MARUYAMA Masao 丸山真男, 1946 《Kindai teki shii » 近代的思惟 (La pensée moderne), Bunka kaigi 文化会議, janvier, $n^{\circ} 1$, Seinen bunka kaigi 青年文化会議.

\section{McNEILL David}

\& ADELSTEIN Jake, 2011

"What Happened at Fukushima? ", The Asia-Pacific Journal, Vol. 9, Issue 32, No 2, 8 août.

\section{NISHIOKA Nobuyuki, 2011}

«Toward a Peaceful Society Without Nuclear Energy: Understanding the Power Structures Behind the 3.11 Fukushima Nuclear Disaster », The AsiaPacific Journal, vol. 9, issue 52, $\mathrm{n}^{\circ} 2$, 26 December.

\section{ŌE Kenzaburō, 1999}

"The Language of Maruyama Masao », in On Politics and Literature. Two Lectures by Kenzaburo Oe, Berkeley, Doreen Townsend Center Occasional Papers, U.C. Berkeley, pp. 5-23.

\section{RIEU Alain-Marc, 1998} «Modernisation : démocratisation et individualisation. Le cas du Japon », in RIEU A.-M. \& ANTOINE A., Individualisme et démocratie : France, 
Etats-Unis, Japon, Lyon, revue Circé, numéro spécial, mai : 55-72.

RIEU Alain-Marc, 2001

Savoir et pouvoir dans la modernisation du Japon, Paris, P.U.F.

ScienceWise, 2011

International Comparison of Public

Dialogue.

SHIOKURA Yutaka, 2011

«Comment un pays irradié est devenu pronucléaire ", Courrier international, 23 août [traduit de l'article publié dans l'Asahi shinbun du 18 août 2011].

TAKEUCHI Yoshimi, 2005

«What is Modernity? (The Case of Japan and China) ", in What is Modernity?:

Writings of Takeuchi Yoshimi, édité, traduit et introduit par Richard F. Calichman, New York, Columbia University Press. 